\title{
Executive function in patients with obstructive sleep apnea treated with continuous positive airway pressure
}

\author{
ESTHER YUET YING LAU, ${ }^{1}$ GAIL A. ESKES, $, 2,3,4$ DEBRA L. MORRISON,, 4 \\ MALGORZATA RAJDA, ${ }^{2,5}$ AND KATHLEEN F. SPURR 6 \\ ${ }^{1}$ Department of Psychology, The University of Hong Kong, Hong Kong, China \\ ${ }^{2}$ Department of Psychiatry, Dalhousie University, Halifax, Nova Scotia \\ ${ }^{3}$ Department of Psychology, Dalhousie University, Halifax, Nova Scotia \\ ${ }^{4}$ Department of Medicine, Dalhousie University, Halifax, Nova Scotia \\ ${ }^{5}$ Sleep Clinic and Laboratory, Queen Elizabeth II Health Sciences Centre, Halifax, Nova Scotia \\ ${ }^{6}$ School of Health Sciences, Dalhousie University, Halifax, Nova Scotia
}

(Received November 4, 2009; Final Revision July 15, 2010; AcCePted July 15, 2010)

\begin{abstract}
Obstructive sleep apnea (OSA) is characterized by disrupted breathing and hypoxemia during sleep, daytime sleepiness, and changes in cognition and mood. One important question is regarding the reversibility of cognitive deficits after treatment with continuous positive airway pressure (CPAP). Here, we report the outcomes of CPAP treatment as measured by tests of attention and executive function. Thirty-seven individuals with moderate to severe OSA and compliant on CPAP treatment were studied with working memory tasks, neuropsychological testing, and overnight polysomnographic sleep study and compared to 27 healthy controls. CPAP improved the respiratory disturbance index, minimum and mean oxygen saturation $\left(\mathrm{SpO}_{2}\right)$, subjective sleep quality, and daytime sleepiness ratings compared to pre-treatment values. In terms of current neurocognitive function, treated individuals with OSA performed at a comparable level to controls on basic working memory storage functions but still showed a significant reduction on tests of working memory requiring the central executive. The OSA group also performed worse on neuropsychological measures of complex attention, executive function, and psychomotor speed. While CPAP is an effective treatment for OSA in terms of ameliorating breathing disruption and oxygen desaturation during sleep, as well as daytime sleepiness, some cognitive deficits may be more resistant to treatment. (JINS, 2010, 16, 1077-1088.)
\end{abstract}

Keywords: Executive function, Working memory, Obstructive sleep apnea syndrome, CPAP treatment, Hypoxemia

\section{EFFECTS OF OSA ON EXECUTIVE FUNCTION}

Research in the past 20 years has highlighted numerous neuropsychological deficits associated with obstructive sleep apnea-hypopnea syndrome (OSA) (for review, see Aloia, Arnedt, Davis, Riggs, \& Byrd, 2004; Engleman, Kingshott, Martin, \& Douglas, 2000; Sateia, 2003; Verstraeten, Cluydts, Pevernagie, \& Hoffmann, 2004). Impairment on tests of executive function is frequently reported in studies of individuals with untreated OSA, although the findings are not always consistent. Saunamäki and Jehkonen (2007) reviewed 40 studies, 12 of which included a healthy control group in testing executive function. Deficits were found on tests presumed to measure different aspects of executive

Correspondence and reprint requests to: Esther Yuet Ying Lau, Add: K641, Knowles Building, Department of Psychology, The University of Hong Kong, Pokfulam Road, Hong Kong. E-mail: eyylau@ @ku.hk function including working memory, phonological fluency, cognitive flexibility, and planning.

The impact of OSA on executive function has been questioned, however (Beebe \& Gozal, 2002; Verstraeten et al., 2004), given that most investigations have not distinguished between the executive controller and the underlying basic cognitive abilities upon which the controller is presumed to act. Two recent studies (Lis et al., 2008; Naëgelé, Launois, Mazza, Feuerstein, Pépin, \& Lévy, 2006) have included different tasks in attempting to distinguish between the executive controller and the sub-processes of maintenance and storage (cf. Baddeley, 1996a, 1996b). Naëgelé and colleagues found that an untreated OSA group did not differ from the control group on dual tasks (e.g., auditory digit span combined with visual span) used to measure control of attentional resources, but did differ on transformed auditory span or modified PASAT, measuring the maintenance and processing components of working memory. They concluded that individuals with OSA have difficulties in maintenance 
and processing of new information while their attentionresource allocation was normal. Given that the tasks were not objectively equated and even the basic tasks required manipulation of information held in mind, the differences in the need for the executive controller may have been minimized among tasks. In contrast, Lis and colleagues separated executive function (measured by different levels of the n-back task) from underlying basic processes (measured by simple and choice reaction time, RT). The untreated OSA group showed slower RTs on all tasks but lowered accuracy only on the n-back tasks compared to the healthy control group. The authors concluded that RT slowing in the OSA group was attributed to deficits in more basic cognitive processes while reduced accuracy in working memory tasks indicated executive dysfunction that could not be attributed to basic processes. The question of OSA effects on executive function versus more basic underlying cognitive processes requires more investigation, but these studies do suggest OSA impact on the executive controller may be distinguished from basic processes.

\section{Reversibility of Cognitive Deficits with Treatment}

Continuous positive airway pressure (CPAP) is considered the treatment of choice for moderate to severe OSA but its effectiveness in reversing cognitive deficits varies across different domains and across studies (Aloia et al., 2004; Jones \& Harrison, 2001). In general, most studies report some improvement in tests of attention/vigilance, memory, and executive functioning (Alchanatis, Zias, Deligiorgis, Amfilochiou, Dionellis, \& Orphandou, 2005; Bonnet, 1993; Douglas, 1998; Montplaisir, Bédard, Richer, \& Rouleau, 1992; Sánchez, Buela-Casal, Bermúdez, \& Casas-Maldonado, 2001), although changes are often inconsistent between studies or within a study between tests even within a cognitive domain (e.g., executive function) (Saunamäki \& Jehkonen, 2007; Weaver, 2001). This inconsistency may be due, at least in part, to methodological differences, including differences in patient population, treatment parameters, inclusion of appropriate controls, definition of executive function, as well as the use of repeated testing leading to potential practice effects. Several investigators have highlighted the resistance of executive dysfunction to CPAP treatment and proposed that because these changes appear more related to the symptoms of hypoxia, executive dysfunction reflects the underlying more permanent pathophysiological damage due to hypoxemia (Bédard, Montplaisir, Malo, Richer, \& Rouleau, 1993; Ferini-Strambi et al., 2003; Feuerstein, Naëgelé, Pepin, \& Levy, 1997; Naëgelé, Pepin, Levy, Bonnet, Pellat, \& Feuerstein, 1998; Naëgelé et al., 1995) (see Table 1 for a summary). All of these studies, however, tested the OSA group twice and the control group only once at baseline, making their results post-treatment susceptible to the confounding effects of practice.

To summarize the findings on reversibility of executive deficits, improvements were found in cognitive flexibility, speed, and non-verbal planning, based on five studies that have a healthy control group at baseline (Saunamäki \& Jehkonen, 2007). However, the residual deficits reported were inconsistent across these studies even when the same tests were used (Table 1).

Interpretation of these studies is also made difficult due to general methodological issues including the lack of control tests for basic components of capacity and/or speed in performance contained in executive tasks and the potential confounding of presumed treatment effects due to practice from repeated testing of participants. In addition, comparing CPAP treatment with placebo or conservative treatment, only three out of nine studies found significant improvements in executive tests in the CPAP group (Barnes et al., 2002; Engleman, Martin, Deary, \& Douglas, 1994, 1997). Taken together, the literature to date suggests that deficits in executive function such as working memory can persist after CPAP treatment (Saunamäki \& Jehkonen, 2007). Confirmation of these findings is needed, however, in studies that use measures to differentiate the executive aspects versus the basic underlying processes confounded in any standardized measure of executive function. In addition, valid comparisons to a healthy control group and avoidance of repeated testing are necessary to draw meaningful conclusions about the residual cognitive deficits of individuals with OSA after treatment.

\section{Investigation of Executive Function Using a Working Memory Model}

This study investigated the executive function of individuals with OSA treated with CPAP using the working memory model developed by Baddeley and colleagues (Baddeley, 1996a, 1996b; Baddeley \& Della Sala, 1996) that allows the dissociation of an executive controller from basic modality specific processing functions as recommended in previous reviews (Beebe \& Gozal, 2002; Verstraeten et al., 2004). In this model, working memory is conceived as based on a supra-modality supervisory (executive) attention system that controls the processes of two basic underlying systems involved in the temporary maintenance and processing of verbal material (called the phonological loop), and visual/ spatial information (visuospatial sketchpad), as well as an episodic buffer that provides a limited capacity multi-modal interface between systems (Baddeley, 2003). Investigating the impact of OSA on executive function and associated subsystems is critical for the evaluation of treatment outcome and the development of appropriate cognitive rehabilitation programs (Levine et al., 2000; Sturm, Willmes, Orgass, \& Hartje, 1997). An understanding of the cognitive deficits may also help elucidate associated neuropathological mechanisms that respond differentially to treatment.

We chose to compare treated patients to age- and educationmatched healthy controls without pre-treatment neurocognitive testing because executive function is highly susceptible to learning and practice effects (Décary, Rouleau, \& Montplaisir, 2000) and repeated testing can essentially invalidate these tests because they are designed to measure behaviors under 
Table 1. Summary of studies on reversibility of executive deficits in OSA after CPAP with a healthy control group

\begin{tabular}{|c|c|c|c|c|c|}
\hline Citation & OSA group & Controls (at baseline) & $\begin{array}{l}\text { Treatment } \\
\text { duration }\end{array}$ & $\begin{array}{l}\text { Post-treatment executive } \\
\text { functions (persistent } \\
\text { deficits in bold) }\end{array}$ & $\begin{array}{l}\text { Conclusions drawn } \\
\text { by authors }\end{array}$ \\
\hline $\begin{array}{l}\text { Bédard et al., } \\
1993\end{array}$ & $\begin{array}{l}N=10 \text { moderate } \\
\text { to severe OSA, } \\
\text { all males }\end{array}$ & $\begin{array}{l}N=10, \text { age- and } \\
\quad \text { education-matched, } \\
\quad \text { all males }\end{array}$ & 6 to 10 months & $\begin{array}{l}\text { Trail Making Test-B } \\
\text { Fluency-p } \\
\text { Rey-Osterreith Complex } \\
\text { Figure Test }(\text { Copy })^{=H C} \text {, } \\
\text { Mazes }^{+}\end{array}$ & $\begin{array}{l}\text { Persistence of } \\
\text { executive deficits } \\
\text { may be associated } \\
\text { with severity of } \\
\text { nocturnal hypoxemia }\end{array}$ \\
\hline $\begin{array}{l}\text { Ferini-Strambi } \\
\text { et al., } 2003\end{array}$ & $\begin{array}{l}N=23 \text { severe OSA, } \\
21 \text { males }\end{array}$ & $\begin{array}{l}N=23 \text { age- and } \\
\text { education-matched, } \\
19 \text { males }\end{array}$ & 4 months & $\begin{array}{l}\text { Digit Span-Forward }{ }^{\mathrm{N}} \text {, } \\
\text { Digit Span-Backward }{ }^{\mathrm{N}} \text {, } \\
\text { Corsi's block-tapping } \\
\text { test }^{\mathrm{N}} \text {, Trail Making } \\
\text { Test-B }^{\mathrm{N}} \text {, Stroop- } \\
\text { performance time }^{\mathrm{N}} \text {, } \\
\text { Stroop-errors } \\
\text { Phonological fluency } \\
\text { }{ }^{\mathrm{N}} \text {, } \\
\text { Rey-Osterreith Complex } \\
\text { Figure Test (Copy) } \\
\text { Raven's progressive }^{\mathbf{0}} \\
\text { matrices }^{\mathbf{0}}\end{array}$ & $\begin{array}{l}\text { Nocturnal hypoxemia } \\
\text { correlated with } \\
\text { pre-treatment } \\
\text { performances on } \\
\text { Fluency-p and } \\
\text { ROCFT }\end{array}$ \\
\hline $\begin{array}{l}\text { Feuerstein et al., } \\
1997\end{array}$ & $\begin{array}{l}N=10 \text { moderate to } \\
\text { severe OSA } \\
\text { all males }\end{array}$ & $\begin{array}{c}N=10 \text { age-, verbal } \\
\text { IQ-, and education- } \\
\text { matched, all males }\end{array}$ & 4 to 6 months & $\begin{array}{l}\text { Digit Span-Forward }{ }^{\mathbf{0}} \text {, } \\
\text { Digit Span-Backward }^{\mathbf{0}} \text {, } \\
\text { Corsi' block-tapping } \\
\text { test }^{\mathbf{0}} \text {, Double encoding } \\
\text { task }^{\mathbf{0}} \text {, Trail Making } \\
\text { Test-Trail B }{ }^{\mathrm{N}} \text {, Stroop- } \\
\text { performance time }=\mathrm{HC}, \\
\text { Wisconsin Card Sorting } \\
\text { Test-number of } \\
\text { categories }^{\mathrm{N}} \text {, Wisconsin } \\
\text { Card Sorting Test- } \\
\text { perseverative errors }=\mathrm{HC}, \\
\text { Phonological fluency } \mathrm{N}\end{array}$ & $\begin{array}{l}\text { Persistent executive } \\
\text { deficits manifested } \\
\text { as difficulties in } \\
\text { acquisition of } \\
\text { information for } \\
\text { memory processing }\end{array}$ \\
\hline $\begin{array}{l}\text { Naëgelé et al., } \\
1998\end{array}$ & $\begin{array}{l}N=10 \text { moderate } \\
\text { to severe OSA, } \\
\text { all males }\end{array}$ & $\begin{array}{c}N=10 \text { age- }, \text { verbal } \\
\text { IQ-, and education- } \\
\text { matched, all males }\end{array}$ & 4 to 6 months & 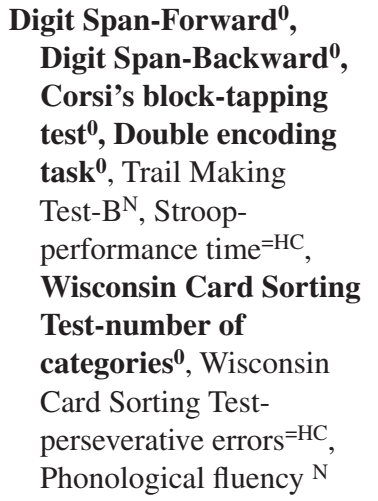 & $\begin{array}{l}\text { Persistent impairments } \\
\text { may be related to } \\
\text { frontal dysfunction, } \\
\text { potentially } \\
\text { associated with } \\
\text { pre-treatment } \\
\text { hypoxemia and sleep } \\
\text { fragmentation }\end{array}$ \\
\hline $\begin{array}{l}\text { Thomas et al., } \\
2005\end{array}$ & $\begin{array}{l}N=16 \text { severe OSA } \\
15 \text { males }\end{array}$ & $\begin{array}{l}N=16 \text { healthy } \\
\text { controls, } 11 \text { males }\end{array}$ & 6 weeks & Verbal 2-back Task ${ }^{0}$ & $\begin{array}{l}\text { Working memory may } \\
\text { be impaired and may } \\
\text { be associated with } \\
\text { lowered activation in } \\
\text { dorsolateral } \\
\text { prefrontal cortex }\end{array}$ \\
\hline
\end{tabular}

Note. "N" indicates that performance was within normal limit at pre-treatment; "0" indicates no change in performance in post-treatment as compared to pre-treatment; " + " indicates significant improvements in performance in post-treatment as compared to pre-treatment; "=HC" indicates that performance was comparable to healthy control group.

conditions of novelty and strategic planning. With repeated testing, these measures may lose sensitivity as executive function tests (Jones \& Harrison, 2001), despite intensive familiarization efforts (Engleman et al., 1994). In addition, to ensure that the patients were indeed properly treated with CPAP, we compared data on their post-treatment sleepiness, 
sleep quality and polysomnographic status [i.e., sleepiness scores, subjective sleep quality, polysomnography (PSG) data] to clinically obtained pre-treatment data.

We hypothesized that: (1) The performance of the stably treated OSA group would be comparable to healthy controls on tests of basic storage and rehearsal components in working memory; (2) In contrast, the stably treated OSA group would show worse performance than healthy controls on tests that demand the involvement of the central executive; (3) The OSA group would show comparable performance to controls on neuropsychological testing except for executive function.

\section{METHODS}

\section{Participants}

The OSA group was recruited based on inclusion criteria of: (1) previous diagnosis of moderate to severe OSA [respiratory disturbance index (RDI) > 15]; (2) treatment with CPAP for at least three months with compliance of at least four hours per night for $80 \%$ of the week (self report was verified by build-in smart card for $73 \%$ of patients). Exclusion criteria included: (1) Concurrent diagnoses of other sleep pathologies or significant medical or neurological conditions; (2) Current alcohol or drug abuse according to the Diagnostic and Statistical Manual of Mental Disorders, Fourth Edition, Text Revision (American Psychiatric Association, 2000); (3) Current formally diagnosed psychiatric illness with onset before diagnosis of sleep apnea; (4) Current use of medication that could affect cognitive function; (5) Current OSA treatment other than CPAP. Age- and education-matched controls with no sleep disorders as verified by overnight PSG were recruited from the community.

\section{Measures}

\section{Sleepiness}

The Epworth Sleepiness Scale (ESS, Johns, 1991, 1992) was used to measure daytime sleepiness. A score greater than 10 (of 24) indicates excessive sleepiness (Hartenbaum et al., 2006).

\section{Sleep Quality}

The Pittsburg Sleep Quality Inventory (PSQI, Buysse, Reynolds, Monk, Berman, \& Kupfer, 1989) measured sleep quality and disturbances over a 1-month time interval. A score $>5$ (of 21) indicates poor sleep.

\section{Neuropsychological Assessment}

A comprehensive neuropsychological test battery was administered, including tests recommended by Décary et al. (2000). The battery included standardized tests covering attentional and executive functioning, immediate and longdelayed recall, and a global evaluation of intellectual functioning (see Table 5 for the list of tests).

\section{Working Memory: Phonological Loop}

Digit span. Verbal storage in the phonological loop was measured by verbal recall for item lists of increasing length (Della Sala, Baddeley, Papagno, \& Spinnler, 1995). The Digit Span was the longest list length for which the participant recalled at least five of six lists correctly.

Verbal memory scanning task. As adapted from Sternberg (1966, 1969), each trial had four sequential events: (1) a fixation cross; (2) simultaneous presentation of a memory set of 2, 4, or 6 uppercase letters randomly chosen from a pool of 12 consonants; (3) a retention interval for $3000 \mathrm{~ms}$; and (4) a single lowercase probe target letter, which stayed on the screen until the participant indicated whether the probe was on the just-presented list by pressing one of two buttons. Accuracy and RTs were recorded. Half of the trials were matches.

\section{Working Memory: Visuospatial Sketchpad}

Visual span. The visual-spatial memory span was measured by the forward span of the Visual Span subtask of the Wechsler Memory Scale-Revised (WMS-R) (Wechsler, 1987).

Spatial memory scanning task. As adopted from the method of Gevins et al. (1996), Smith, Jonides, Koeppe, Awh, Schumacher, and Minoshima (1995), and Smith, Jonides, and Koeppe (1995), each trial had four sequential events: (1) a fixation cross; (2) a set of 2, 4, or 6 target dots arrayed on 12 possible positions, each of which was randomly generated within a donut-shaped area between 1.5 and 4.5 degree of radius from the screen's centre; (3) a retention interval for $3000 \mathrm{~ms}$; (4) a location probe of a single outline target circle that remained on the screen until the viewer responded as to whether the circle indicated a previously viewed location by pressing one of two buttons. Accuracy and RTs were recorded. Half of the trials were matches.

\section{Working Memory: Central Executive}

$\mathrm{N}$-back task. This continuous memory task was adapted by Smith et al. (1996), from the one developed by Gevins and Cutillo (1993). In both verbal and spatial conditions, subjects were presented a continuous stream of single letters appearing at a randomly chosen location amongst 12 possible positions, which matched with the memory scanning tasks. Both the position and identity of the letters varied on each trial, with the only difference between verbal and spatial tasks being the response requirement (letter identity $v s$. spatial location).

In the 2-back condition, subjects had to decide whether or not each letter matched in identity (verbal) or in location (spatial) the item presented two back. In each block, 10/26 trials were matches, with four foils at the 1-back position. Accuracy and RTs were recorded. 
In the 0 -back control condition, a similar sequence of letters as in the 2-back condition was presented, but now the task was to decide whether or not each letter matched a single target letter or spatial location specified at the beginning of each block. The number of matches in the 0-back conditions was equivalent to the 2 -back conditions.

Working Memory Span (Episodic Buffer). This task measures the capacity to hold and manipulate information in long-term memory, a process calling upon the episodic buffer of the central executive. The subjects were presented with a series of sentences and asked to verify them as probable or not, as well as to recall the last word of each sentence in the order of presentation at the end of the series (Daneman \& Carpenter, 1980).

Performance on each sub-task (i.e., Word Span and Sentence Verification Span) was assessed so that basic capacity could be taken into account when the tasks were combined in the Working Memory Span dual task (Duff \& Logie, 2001). Three lists of one-syllable nouns to be used as the last words to be recalled in each sentence were generated from the Medical Research Council (MRC) Psycholinguistic Database: Machine Usable Dictionary, Version 2 (Coltheart, 1981a, 1981b; Wilson, 1988) and balanced for their concreteness and familiarity ratings.

Word span.-Performance was assessed by providing subjects with item lists of increasing length and asking for immediate recall. Three trials at each list length were given. Each word was presented for $2 \mathrm{~s}$ on a computer screen, with an inter-stimulus-interval of $0.5 \mathrm{~s}$. At the end of each list, recall was cued by a tone and audio-recorded. The test continued until the participant failed to recall two of three trials at a list length. The total number of word lists correctly recalled before the test was discontinued, the proportion of trials correctly recalled, and the Word Span, which was the longest list length reached were recorded.

Sentence verification span.-Participants were asked to judge the plausibility of sentences (overall probability of plausible/implausible sentences $=50 / 50$ ) of three to six words, one at a time on a computer screen starting with two sentences in a list and increasing up to 10 . Three trials at each list length were presented. Each list of sentences was presented at a predetermined rate over $10 \mathrm{~s}$, so that at longer list lengths, sentences were presented more rapidly. List length increased by one until the participant could no longer correctly verify all of the sentences on two out of three trials for a given list length. Sentence verification span was the longest list length completed.

Working memory span.-For the dual task procedure, participants were asked to both verify sentences and to memorize the last word of each sentence for serial recall at the end of the sentence list. Outcome measures were the same as in the single tasks.

\section{Procedures}

The protocol was prepared in accordance with the Helsinki Declaration and was approved by the Research Ethics Board of the Queen Elizabeth II Health Sciences Centre, Halifax, Nova Scotia. OSA participants were recruited by invitation letters to all patients of the Sleep Disorders Laboratory of the Queen Elizabeth II Health Sciences Centre. After an initial screening and consenting procedure, participants filled out questionnaires of subjective sleep quality, sleepiness, and psychosocial measures (findings reported in another study under preparation). Participants then completed the working memory experimental tasks and the neuropsychological battery. All participants also received an overnight PSG using standardized procedures and scoring within eight weeks of the cognitive testing (Rechtschaffen \& Kales, 1968). An obstructive apnea was defined as airflow cessation of at least $10 \mathrm{~s}$ in the presence of continued respiratory effort (Kushida et al., 2005). An obstructive hypopnea was defined as a reduction in airflow of at least $50 \%$ of biocalibration, lasting a minimum of $10 \mathrm{~s}$ followed by an EEG arousal and/ or fall in oxygen saturation of at least $4 \%$. RDI was calculated as: total number of apneas and hypopneas/total sleep time (hr). For the OSA group, relevant health information was extracted from patients' health charts at the Sleep Laboratory, including history of OSA, other relevant medical history and medications, and pretreatment clinical and PSG data.

\section{RESULTS}

\section{Participants' Characteristics}

Data from 37 individuals with OSA and 27 healthy controls were included in the analyses. Seven control participants were excluded because of elevated RDI seen in the sleep study (RDI > 15). Eight controls (30\%) and two treated OSA participants (18\%) had a RDI of 5-15. It should be noted that none of these control participants displayed daytime symptoms of OSA and did not meet clinical criteria for diagnosis of OSA and thus were included in the study.

There were no significant differences between the OSA group and the control group on age, $t(62)=-.48$ and education level, $t(62)=.61$ but, as expected, the mean body mass index (BMI) of the OSA group was significantly higher than that of the control group, $t(62)=-4.83 ; p<.001$ (Table 2). Stepwise regressions showed that BMI was not a significant predictor of any working memory task and therefore did not contribute to any group differences reported below. Gender was found to be a significant predictor of only Verbal 0-back accuracy, with males $(M=98.76 \% ; S D=1.29 \%)$ having better performance than females $(M=97.51 ; S D=2.83)$, $R^{2}=.073$, beta $=.27, p=.03$. Clinical information for the OSA group pre- and post-treatment is also presented in Table 2 .

\section{Pre- and Post-Treatment Within-Group Comparisons}

Compared to pre-treatment clinical evaluation, individuals with OSA treated with CPAP showed significant improvements 
Table 2. Group demographic characteristics and OSA group medical history

\begin{tabular}{|c|c|c|c|c|}
\hline & OSA group $(N=37)$ & Range & Controls $(N=27)$ & Range \\
\hline Age (years) & $57.9(9.5)^{\mathrm{a}}$ & $40-76$ & $56.7(10.5)^{\mathrm{a}}$ & $33-85$ \\
\hline $\operatorname{Sex}(F: M)$ & $15: 22$ & & 19: 8 & \\
\hline Handedness (R: L) & $34: 3$ & & 25: 2 & \\
\hline Education (years) & $15.1(3.6)^{\mathrm{a}}$ & $10-24$ & $15.7(3.2)^{\mathrm{a}}$ & $11-23$ \\
\hline BMI & $33.5(7.4)^{\mathrm{a}}$ & $24.0-56.6$ & $25.5(5.0)^{\mathrm{a}}$ & $18.1-38.0$ \\
\hline Time since onset of OSA symptoms (years) & $13.6(9.7)^{\mathrm{a}}$ & $2-53$ & & \\
\hline Time since diagnosis of OSA (months) & $25.6(21.1)$ & $6-120$ & & \\
\hline Duration CPAP treatment (months) & $17.8(11.4)$ & $3-47$ & & \\
\hline Usage of CPAP per week (hours) & $51.4(6.7)$ & $35-70$ & & \\
\hline CPAP compliance ${ }^{b}$ & $96.1(5.6)$ & $81.0-100$ & & \\
\hline
\end{tabular}

Note . $\mathrm{BMI}=$ body mass index OSA = obstructive sleep apnea; $\mathrm{CPCP}=$ continuous positive airway pressure

${ }^{\mathrm{a}}$ Mean $(S D)$.

bPercentage of days with usage $>4$ hours in the last 3 months. For $73 \%$ of the participants, compliance was determined objectively by downloading data from the build-in smart card of their CPAP machines. Self report of usage was used for the rest of the participants whose machines were not equipped with a smart card.

on respiratory and oxygen saturation indexes (RDI, minimum $\mathrm{SpO}_{2}$, mean $\mathrm{SpO}_{2}$ ), sleepiness (ESS), and sleep quality (PSQI), and showed comparable scores with the control group (Table 3).

\section{Between-Group Comparisons}

\section{Working memory tasks}

Phonological loop. Analysis of variance (ANOVA) was used to analyze the modified digit span and the Verbal Memory Scanning Task (Table 4). No significant group differences were shown on these measures.

Visuo-spatial sketchpad. Parallel analyses were conducted on the Spatial Span (Forward) and the Spatial Memory Scanning Task (Table 4), and no significant differences were found.

Central executive: $n$-back task. RT and accuracy data were each analyzed using two-way mixed factorial ANOVAs with 2 Groups (OSA vs. controls) * 2 Task Conditions (0-back vs. 2-back) for the verbal and spatial tasks separately. For the Verbal Task, the main effect of Task Condition was significant for both RT and accuracy data, with longer RTs in the 2-back condition compared to the 0-back condition, $\mathrm{F}(1,62)=$ $255.61 ; \mathrm{p}<.001$ and with lower accuracies on the 2-back compared with the 0 -back condition, $\mathrm{F}(1,62)=96.65 ; \mathrm{p}<.001$. The Task Condition * Group interaction for accuracy data approached significance $(F(1,62)=3.30 ; \mathrm{p}=.074)$, suggesting a trend for a bigger difference between the 0-back and 2-back conditions for patients than for controls. Given our a priori hypotheses about the OSA group performance, we conducted follow-up comparisons of the accuracy of each group on each verbal task. While the two groups did not show significant differences on Verbal 0-back accuracy $\left(M_{\mathrm{OSA}}=97.9 ; \mathrm{SD}=2.5 ; M_{\mathrm{HC}}=98.4 ; \mathrm{SD}=2.1\right)$, the OSA group had a significantly lower accuracy in the 2-back condition $\left(M_{\mathrm{OSA}}=75.8 ; \mathrm{SD}=17.6 ; M_{\mathrm{HC}}=83.6 ; \mathrm{SD}=10.9\right)$, $\mathrm{t}(62)=2.17 ; \mathrm{p}=.03)($ Table 4).

A similar pattern was seen for the Spatial N-back Task. There were significant main effects of Task Condition on both RTs and accuracy data, with slower RTs $(F(1,61)=240.7$;

Table 3. Pre- and post-treatment comparisons of hypoxemia indices, sleepiness, and subjective sleep quality

\begin{tabular}{|c|c|c|c|c|c|c|c|}
\hline & \multirow{2}{*}{$\begin{array}{c}\begin{array}{l}\text { OSA group } \\
\text { post-CPAP }\end{array} \\
(\mathrm{A}) \\
(N=37)\end{array}$} & \multirow{2}{*}{$\begin{array}{c}\begin{array}{c}\text { OSA group } \\
\text { pre-CPAP }\end{array} \\
(\mathrm{B}) \\
(N=37)\end{array}$} & \multirow{2}{*}{$\begin{array}{c}\text { Controls } \\
(\mathrm{C}) \\
(N=27)\end{array}$} & \multicolumn{2}{|c|}{ A vs. B } & \multicolumn{2}{|c|}{ B vs. C } \\
\hline & & & & $t(d f)$ & $p$ & $t(d f)$ & $p$ \\
\hline RDI & $42.2(24.9)^{\mathrm{a}}$ & $1.7(1.5)$ & $4.0(3.4)$ & $9.52(32)$ & $.000 * *$ & $3.25(62)$ & $.003^{\mathrm{b} * *}$ \\
\hline Minimum $\mathrm{SpO}_{2}$ & $80.2(9.8)$ & $90.3(3.6)$ & $88.6(3.3)$ & $5.61(27)$ & $.000 * *$ & $1.90(60)$ & .062 \\
\hline Mean $\mathrm{SpO}_{2}$ & $93.7(3.5)$ & $95.7(1.6)$ & $95.7(0.9)$ & 3.17 (29) & $.004 * *$ & $.098(62)$ & .923 \\
\hline ESS & $14.4(5.2)$ & $8.3(4.5)$ & $6.6(4.7)$ & $7.52(36)$ & $.000 * *$ & $1.48(62)$ & .144 \\
\hline PSQI Global Score & $8.5(3.3)$ & $4.4(2.4)$ & $4.6(2.8)$ & $5.85(27)$ & $.000 * *$ & $0.20(62)$ & .776 \\
\hline
\end{tabular}

Note. $\mathrm{RDI}=$ respiratory disturbance index $; \mathrm{SpO}_{2}=$ peripheral oxygen saturation; $\mathrm{ESS}=$ Epworth Sleepiness Scale; PSIQ $=$ Pittsburg Sleep Quality Index; CPAP = continuous positive airway pressure; OSA = obstructive sleep apnea.

aMean $(S D)$.

${ }^{\mathrm{b}} \mathrm{OSA}$ Group has lower RDI after treatment as compared with healthy controls.

$* * p<.01$. 
Table 4. Working memory task performance of the treated OSA group and the control group

\begin{tabular}{|c|c|c|c|}
\hline & OSA group $(N=37)$ & Controls $(N=27)$ & Effect size $(d)$ \\
\hline \multicolumn{4}{|l|}{ Phonological Loop } \\
\hline Modified Digit Span & $5.9(1.3)$ & $6.0(1.2)$ & -0.08 \\
\hline \multicolumn{4}{|l|}{ Verbal Memory Scanning } \\
\hline Reaction Time (ms) & $1300.8(207.7)^{\mathrm{a}}$ & $1319.1(273.9)$ & -0.07 \\
\hline Accuracy (\% correct) & $89.5(5.8)$ & $89.8(5.3)$ & -0.06 \\
\hline \multicolumn{4}{|l|}{ Verbal 0-back } \\
\hline Reaction Time (ms) & $707.4(121.7)$ & $635.7(96.7)^{*}$ & 0.74 \\
\hline Accuracy $(\%)$ & $97.9(2.5)$ & $98.4(2.1)$ & -0.24 \\
\hline \multicolumn{4}{|l|}{ Visuo-spatial Sketchpad } \\
\hline Spatial Span & $8.1(1.8)$ & $9.3(1.9)$ & -0.63 \\
\hline \multicolumn{4}{|l|}{ Spatial Memory Scanning } \\
\hline Reaction Time (ms) & $1316.8(286.0)^{\mathrm{a}}$ & $1232.5(295.4)$ & 0.29 \\
\hline Accuracies (\% correct) & $82.0(5.6)$ & $84.5(5.9)$ & -0.42 \\
\hline \multicolumn{4}{|l|}{ Spatial 0-back } \\
\hline Reaction Time (ms) & $748.3(128.6)$ & $715.3(155.7)$ & 0.21 \\
\hline Accuracies $(\%)$ & $92.4(2.8)$ & $92.1(3.6)$ & 0.08 \\
\hline \multicolumn{4}{|l|}{ Central Executive } \\
\hline \multicolumn{4}{|l|}{ Verbal 2-back } \\
\hline Reaction Time (ms) & $1219.9(234.9)$ & $1213.4(270.8)$ & 0.02 \\
\hline Accuracies (\%) & $75.8(17.6)$ & $83.6(10.9)^{*}$ & -0.72 \\
\hline \multicolumn{4}{|l|}{ Spatial 2-back } \\
\hline Reaction Time (ms) & $1153.6(225.6)$ & $1084.5(229.6)$ & 0.30 \\
\hline Accuracies $(\%)$ & $78.0(16.9)$ & $87.1(8.7)^{* *}$ & -1.05 \\
\hline Working Memory Span & $2.9(1.0)^{\mathrm{a}}$ & $3.3(1.0)$ & -0.4 \\
\hline \multicolumn{4}{|l|}{ Single Task Condition } \\
\hline Word Span & $4.7(1.0)$ & $5.1(1.0)$ & -0.4 \\
\hline Proportion of Words Correctly Recalled & $.83(.07)$ & $.83(.06)$ & 0 \\
\hline Sentence Verification Span & $3.8(1.4)$ & $4.2(1.5)$ & -0.27 \\
\hline Proportion of Sentences Correctly Verified & $.74(.11)$ & $.78(.12)$ & -0.33 \\
\hline \multicolumn{4}{|l|}{ Dual Task Condition } \\
\hline Word Span & $3.0(1.0)$ & $3.4(1.0)$ & -0.4 \\
\hline Proportion of Words Correctly Recalled & $.56(.13)$ & $.60(.13)$ & -0.31 \\
\hline Sentence Verification Span & $3.5(1.2)$ & $4.1(1.1)^{*}$ & -0.55 \\
\hline Proportion of Sentences Correctly Verified & $.68(.10)$ & $.78(.10) * *$ & -1 \\
\hline
\end{tabular}

aMean $(S D)$.

$* p<.05$.

$* * p<.01$

$p<.001)$ and lower accuracies $(F(1,61)=34.13 ; p<.001)$ for the 2-back condition compared to the 0-back condition. Accuracy data showed a significant Task Condition * Group interaction effect $(F(1,61)=9.68 ; p<.01)$, and follow-up comparisons indicated that while OSA group and controls did not differ on the 0 -back condition $(t(62)=0.34 ; p=.74)$, patients were less accurate than controls in the 2-back condition $(t(62)=2.81 ; p<.01)($ Table 4$)($ Figure 1$)$.

Central Executive: Working memory span. The total number and proportion of words recalled (Word Span) and sentences correctly verified (Sentence Verification Span), were each analyzed using 2 Groups (OSA vs. controls) $* 2$ Task Conditions (single vs. dual) ANOVAs (Table 4). A significant interaction between Task Condition and Group $(\mathrm{F}(1,62)=5.54 ; \mathrm{p}=.02)$ was seen, and follow-up $\mathrm{t}$ tests showed that while the two groups did not differ on any measure when they performed the two tasks separately, the proportion of correctly verified sentences in the dual condition was significantly smaller in patients $(M=.68$; $\mathrm{SD}=.10)$ compared to controls $(\mathrm{M}=.78 ; \mathrm{SD}=.10 ;(\mathrm{t}(62)=$ $3.95 ; \mathrm{p}=<.01$, Figure 2).

\section{Neuropsychological Tests}

The OSA group and controls were compared on their performance on the neuropsychological battery (Table 5). Four measures that have several levels (i.e., Consonant Trigrams, Trail Making, Digit Span (WAIS-R), and Spatial Span (WMS-R)) were analyzed using mixed factorial ANOVAs; other neuropsychological tests were analyzed using independent samples $t$ tests. Worse performance by the OSA group was seen on Vocabulary $(p<.05)$, measures of attention and concentration $\left(\mathrm{D}_{2}\right.$ Test of Attention, Digit Symbol, $p<.01$; and PVT lapses, $p<.05$ ), as well as on tests of executive function (Stroop interference score, Wisconsin Cord 


\section{Spatial N-back Task - Accuracies}

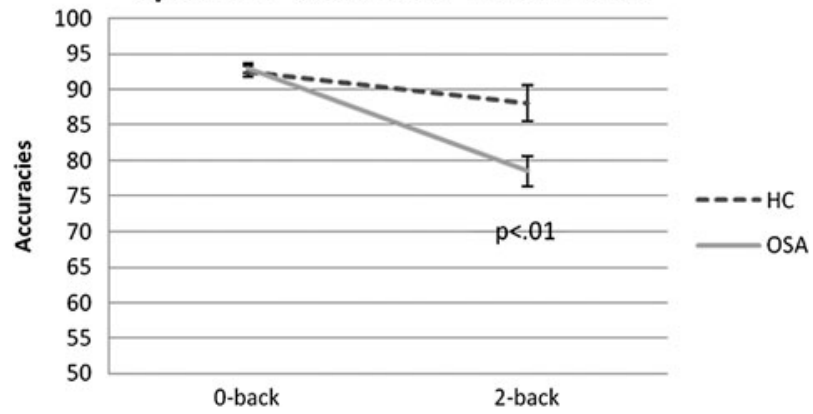

Fig. 1. Interaction effects between task condition and group on the Spatial N-back Task. The accuracy of the obstructive sleep apnea (OSA) group deteriorated disproportionately in the 2-back condition as compared to the healthy control group. Error bars represent standard error of each data point.

Sorting Test perseverative and non-perseverative errors, $p<.01$; WCST categories and Trail Making B; $p<.05$ ), and psychomotor dexterity and speed (Grooved Pegboarddominant hand; $p<.01$ ).

Follow-up regression analyses were conducted to explore underlying cognitive processes associated with working memory deficits shown in the OSA group. First, as there were significant between group differences on the Vocabulary raw score, all working memory measures were regressed on Vocabulary after entering demographic variables of age, education, and gender. It was found that Vocabulary, together with the demographic variables significantly predicted Verbal 0 -back RT $\left(R^{2}=.53 ; R^{2}\right.$ change $=.21 ;$ beta $\left.=-0.53 ; p<.001\right)$, as well as the proportion of sentences correctly verified in the dual condition of the Working Memory Span task $\left(R^{2}=.53 ; R^{2}\right.$ change $=.13 ;$ beta $\left.=.41 ; p=.002\right)$. To further understand whether group differences in Vocabulary accounted for the working memory deficits in the OSA group, Vocabulary was used as a covariate in comparing the OSA and the control groups on these two outcome measures. Between group differences on Verbal 0-back RT were no

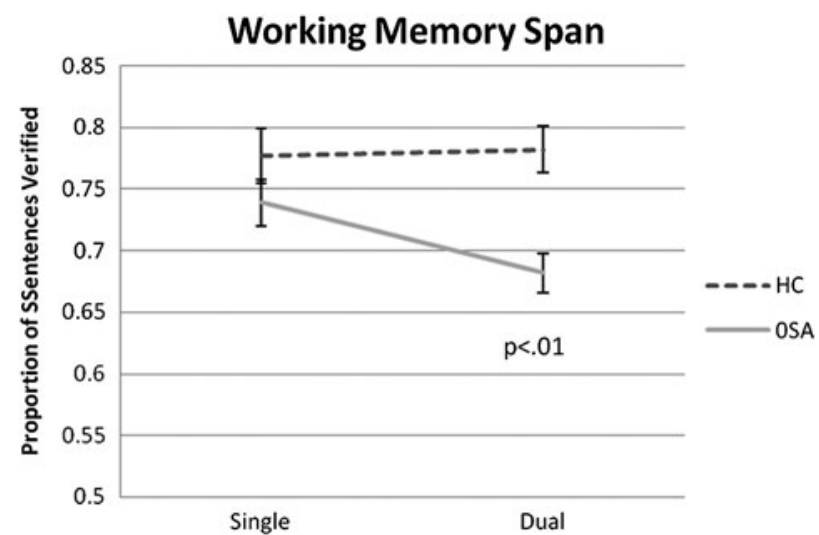

Fig. 2. Interaction effects between task condition and group on working memory span. The proportion of sentences verified by the obstructive sleep apnea (OSA) group deteriorated disproportionately in the dual condition as compared to the healthy control group. Error bars represent standard error of each data point. longer significant, with the effects of Vocabulary covaried out $(F(1)=2.12 ; p=.10)$. On the contrary, there was still a significant main effect of Group on the Proportion of Sentences Verified-Dual Condition as shown in analysis of covariance (ANCOVA), $F(1)=9.81 ; p<.01$.

As the OSA group performed significantly worse than the control group on the Grooved Pegboard task-dominant hand, performances on working memory tasks were regressed on it to investigate if processing speed might be associated with the deficits seen in the OSA group. Above and beyond the variances explained by the demographics, Pegboard performance was associated with Verbal 0-back Accuracy $\left(R^{2}=.18\right.$; $R^{2}$ change $=.08 ;$ beta $\left.=-.39 ; p=.02\right)$, Spatial 2-back Accuracy $\left(R^{2}=.37 ; R^{2}\right.$ change $=.07 ;$ beta $\left.=-.36 ; p=.02\right)$, and the Proportion of Sentences Verified-Dual Condition $\left(R^{2}=.23\right.$; $R^{2}$ change $=.08 ;$ beta $\left.=-.37 ; p=.02\right)$. ANCOVAs showed that after Pegboard performance was taken into account, there were no significant group differences on Verbal 0-back Accuracy, $F(1)=.17 ; p=.68$, and on Spatial 2-back Accuracy, $F(1)=2.35 ; p=.13$, but the OSA group still showed poorer performance on the Proportion of Sentences Verified-Dual Condition, as shown by a significant main effect of Group, $F(1)=11.06 ; p=.002$.

\section{DISCUSSION}

This study aimed at investigating the neurocognitive function of individuals with moderate to severe OSA treated with CPAP. As compared with their pre-treatment status, the patients showed improved sleep (fewer apneas and hypopneas and increased oxygen saturation during sleep), and improved subjective sleep quality and daytime sleepiness, in line with previous studies (Feuerstein et al., 1997; Grunstein, 2005; Kingshott, Vennelle, Hoy, Engleman, Deary, \& Douglas, 2000; Patel, White, Malhotra, Stanchina, \& Ayas, 2003; Sanner, Klewer, Trumm, Randerath, Kreuzer, \& Zidek, 2000). Thus, CPAP treatment was successful in terms of improving OSA symptoms and nighttime sleep in this highly adherent group.

In terms of neurocognitive function, our data support our hypothesis that patients with OSA treated with CPAP perform at a level comparable to healthy controls on neuropsychological tests that are less associated with complex attention and executive function (e.g., list learning, digit span) and on working memory tasks that place less demands on the central executive (e.g., Verbal and Spatial 0-back conditions of the n-back task, and the Single conditions of Word Span and Sentence Verification Span). In contrast to the basic storage and rehearsal function and in support of our hypothesis, the OSA group showed lower accuracy on both the Spatial and Verbal 2-back tasks, as well as on the Sentence Verification Span in the Dual condition of the Working Memory Span task. They also performed worse on several neuropsychological tests requiring complex attention and executive function (D2 Test of Attention, Digit Symbol, Stroop, Wisconsin Card Sorting Test, Trail Making B). 
Table 5. Comparison between the OSA group treated with CPAP and the control group on standardized neuropsychological tests (raw scores)

\begin{tabular}{|c|c|c|c|c|c|}
\hline & OSA group $(N=37)$ & Controls $(N=27)$ & $t(d f=62)$ & $p$ & Effect size $(d)$ \\
\hline \multicolumn{6}{|l|}{ General Intelligence } \\
\hline WAIS-R Vocabulary & $56.6(9.4)^{\mathrm{b}}$ & $61.3(5.9)$ & 2.45 & $.017 *$ & -0.8 \\
\hline WAIS-R Block Design & $30.8(8.3)$ & $33.8(10.1)$ & 1.32 & .193 & -0.3 \\
\hline Estimated FSIQ & $114.6(13.4)$ & $121.0(11.6)$ & 2.00 & .050 & -0.55 \\
\hline \multicolumn{6}{|l|}{ Attention \& Concentration } \\
\hline \multicolumn{6}{|l|}{ D2 Test of Attention ${ }^{\mathrm{a}}$} \\
\hline Total Items & $412.9(90.7)$ & $467.9(80.7)$ & 2.74 & $.008 * *$ & -0.68 \\
\hline Total Errors & $13.9(10.7)$ & $16.7(11.2)$ & 1.02 & .311 & -0.25 \\
\hline Concentration & $157.6(32.7)$ & $185.2(41.6)$ & 2.98 & $.004 * *$ & -0.66 \\
\hline Digit Symbol $^{\mathrm{a}}$ & $53.1(8.0)$ & $60.7(10.4)$ & 3.30 & $.002 * *$ & -0.73 \\
\hline \multicolumn{6}{|l|}{ PVT } \\
\hline Mean RT & $280.3(37.5)$ & $261.4(30.5)$ & 2.09 & .041 & 0.62 \\
\hline Slowest $10 \%$ RRT & $2.4(0.45)$ & $2.61(0.42)$ & 2.06 & .044 & -0.5 \\
\hline Lapses & $2.3(2.5)$ & $1.0(1.8)$ & 2.11 & $.038 *$ & 0.72 \\
\hline WAIS-R Digit Span & $16.1(4.0)$ & $16.9(4.2)$ & 0.72 & .474 & -0.19 \\
\hline Forward & $8.5(2.3)$ & $8.6(2.1)$ & 0.26 & .798 & -0.05 \\
\hline Backward $^{\text {a }}$ & $7.7(2.5)$ & $8.2(2.5)$ & 0.86 & .393 & -0.2 \\
\hline WMS-R Visual Span & $17.1(3.1)$ & $18.0(2.9)$ & 1.17 & .246 & -0.31 \\
\hline Forward & $9.1(1.9)$ & $9.3(1.9)$ & 0.44 & .663 & -0.11 \\
\hline Backward $^{\text {a }}$ & $8.1(1.8)$ & $8.7(1.6)$ & 1.53 & .131 & -0.38 \\
\hline \multicolumn{6}{|l|}{ Memory } \\
\hline \multicolumn{6}{|l|}{ CVLT-II } \\
\hline Trial 1 to 5 Total & $48.6(9.3)$ & $52.7(9.1)$ & 1.74 & .086 & -0.45 \\
\hline S-D Free Recall & $11.1(2.7)$ & $11.1(3.3)$ & 0.08 & .935 & 0 \\
\hline L-D Free Recall & $12.1(2.8)$ & $12.4(2.9)$ & 0.40 & .688 & -0.10 \\
\hline \multicolumn{6}{|l|}{ Rey-O CFT } \\
\hline Copy & $30.7(3.5)$ & $31.3(3.4)$ & 0.68 & .502 & -0.18 \\
\hline Recall & $17.3(6.1)$ & $19.2(6.4)$ & 1.21 & .229 & -0.3 \\
\hline \multicolumn{6}{|l|}{ Executive Function } \\
\hline \multicolumn{6}{|l|}{$\mathrm{CCC}^{\mathrm{a}}$} \\
\hline $9 "$ & $15.0(0.2)$ & $15.0(0.2)$ & .313 & .755 & 0 \\
\hline $18 ”$ & $10.4(2.8)$ & $11.1(2.5)$ & 1.37 & .177 & -0.28 \\
\hline $36 "$ & $9.5(2.7)$ & $10.6(2.4)$ & 1.07 & .290 & -0.46 \\
\hline \multicolumn{6}{|l|}{ WCST $^{\mathrm{a}}$} \\
\hline Categories & $4.8(1.6)$ & $5.6(1.1)$ & 2.26 & $.028 *$ & -0.73 \\
\hline Perseverative Errors & $17.2(11.9)$ & $9.5(9.9)$ & 2.71 & $.009 * *$ & 0.78 \\
\hline Nonperseverative Errors & $16.3(11.5)$ & $7.3(5.5)$ & 3.77 & $.000 * *$ & 1.64 \\
\hline \multicolumn{6}{|l|}{ Trail Making Test } \\
\hline A & $26.9(8.3)$ & $23.6(7.9)$ & 1.76 & .084 & 0.42 \\
\hline $\mathrm{B}^{\mathrm{a}}$ & $74.3(26.1)$ & $61.7(21.5)$ & 2.04 & $.045^{*}$ & 0.59 \\
\hline WISC-III - Mazes ${ }^{\mathrm{a}}$ & $21.6(4.3)$ & $22.9(4.8)$ & 1.05 & .298 & -0.27 \\
\hline \multicolumn{6}{|l|}{ Stroop } \\
\hline Word & $102.6(14.7)$ & $97.7(14.7)$ & 1.31 & .194 & 0.33 \\
\hline Color & $71.9(11.9)$ & $73.0(8.4)$ & 0.42 & .673 & -0.13 \\
\hline Color-Word & $39.0(7.7)$ & $42.5(7.8)$ & 1.77 & .082 & -0.45 \\
\hline Interference $^{a}$ & $-0.91(5.8)$ & $4.2(5.9)$ & 3.43 & $.001 * *$ & -0.87 \\
\hline \multicolumn{6}{|l|}{ Psychomotor speed } \\
\hline \multicolumn{6}{|l|}{ Pegboard } \\
\hline Dominant & $73.0(11.0)$ & $64.7(10.1)$ & 3.04 & $.004 * *$ & 0.82 \\
\hline Non-Dominant & 80.5 (15.6) & $73.8(12.6)$ & 1.82 & .074 & 0.53 \\
\hline
\end{tabular}

Note. WAIS-R, Wechsler Adult Intelligence Scale-Revised; FSIQ, Full Scale Intelligence Quotient; PVT, Psychomotor Vigilance Test; WMS-R, Wechsler Memory Scale-Revised; CVLT, California Verbal Learning Test; S-D, Short delay; L-D, Long Delay; Rey-O CFT, Rey-Osterreith Complex Figure Test; CCC, Brown-Peterson Consonant Trigrams; WCST, Wisconsin Card Sorting Test; WISC-III, Wechsler Intelligence Scale for Children; Pegboard, Grooved Pegboard Test

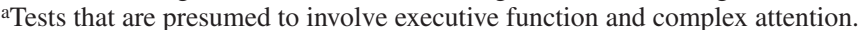

bMean $(S D)$.

$* p<.05$.

$* * p<.01$ 
Our findings suggest that executive difficulties in stably treated OSA are of multiple domains (both verbal and spatial) and beyond the basic functioning of the slave systems of the working memory model. Their deficits were on tasks requiring simultaneous storage and on-line processing and updating of information, and interfacing with long-term memory store. These findings provide support to the stance that difficulties with controlled (complex) attention and executive function in patients with moderate to severe OSA are more chronic despite treatment and may reflect an underlying neuropathology (Bédard et al., 1993; Ferini-Strambi et al., 2003).

The Working Memory Span task is also presumed to require the episodic buffer of the central executive to access the semantic memory for storage (words) and on-line processing (sentence verification) in a dual task. While performance on basic tasks was equivalent between groups, the disproportionate reduction in accuracy in sentence verification in the Dual condition suggests that the OSA group had difficulty maintaining their performance when they were accessing the episodic buffer related to maintaining and rehearsing a series of words in mind. Whether this difficulty in performing a component task during multitasking reflects a deficit of the central executive itself or inefficiency of the episodic buffer is unknown.

These studies confirm and extend the findings of three more recent studies investigating working memory in OSA. Naëgelé and colleagues (2006) studied episodic memory, procedural memory, and working memory and identified maintenance and processing of information as the specific working memory deficit in their untreated patients. However, their tests (i.e., PASAT and self-order spatial memory test) did not have a control condition or a parallel test to control for the basic component processes. Another study that investigated working memory in untreated patients presented findings that were quite consistent with this study (Lis et al., 2008). They found that there were working memory deficits (as seen in decreased accuracies on the n-back task) in untreated patients that were unlikely to be attributable to basic processes. Also consistent with our finding of accuracy deficits, they reported that RT slowing was likely to be associated with more elementary cognitive processes involved in working memory tasks, and deficits in accuracy were more specific to working memory. Up to date, there was only one other study that investigated specifically working memory in patients with OSA post-treatment (FelverGrant, Bruce, Zimmerman, Sweet, Millman, \& Aloia, 2007). They showed that performance on a verbal 2-back task correlated with the other executive function task they used, the PASAT. They also reported that "high treatment adherers" demonstrated post-treatment improvements on a verbal 2-back task and on the PASAT, in comparison to "low adherers." Any conclusion about "improved" performance may be premature, however, given that even baseline pre-treatment levels were within the normal range for all groups and the performance of the comparison group of low adherers actually declined over time.
To examine if demographic differences in BMI and gender between the two groups contributed to the performance differences on working memory tasks, stepwise regression analyses were conducted. BMI was not a predictor of any performance. Gender was found only to be a significant predictor of Verbal 0-back accuracy, with males having better performance. Thus, the group differences in BMI or the inclusion of more males in the OSA group compared to the control group cannot explain the group differences that were found on the main outcome measures of interest, that is, the working memory or executive function tasks.

Regressions and ANCOVAs were used to understand potential underlying cognitive processes involving premorbid ability (Vocabulary) or motor dexterity and processing speed (Grooved Pegboard) that might mediate working memory deficits in the OSA group. Vocabulary scores did not account for group differences that were found on the working memory tasks. For Pegboard, while OSA-related impairment on the task has been reported by previous studies as well (e.g., Kim, Young, Matthews, Weber, Woodard, \& Palta, 1997), its association with working memory has not been examined in studies of OSA. We found that, while changes in motor speed might be related to group differences in some central executive measures (e.g., Spatial 2-back), it was not associated with others (e.g., Verbal 2-back or Proportion of Sentences Verified-Dual Condition on the Working Memory Span). This finding underscores the complexity of mechanisms underlying central executive tasks, and suggests that, at least in some populations, changes in processing speed may be a relevant contributor to more complex deficits. The contribution of processing speed to a variety of cognitive deficits seen in aging has been proposed as well (Salthouse, 1996).

Our study provides evidence for the residual neurocognitive effects of OSA on individuals even after effective treatment with CPAP. Our findings suggest that individuals with more severe OSA tend to perform worse in the long term even when their disorder is treated and their sleep is normalized. We have included individuals with RDI of 5-15 in both OSA and control groups. While these individuals did not present any current daytime symptoms and thus would not qualify for a formal diagnosis of OSA, further research is needed to investigate whether cognitive functioning in asymptomatic and milder forms of OSA is normal with and without treatment. It would also be valuable to explore and validate treatment targeting cognitive functioning to compliment the treatment of OSA itself.

\section{ACKNOWLEDGMENTS}

This study was funded by a Health Research Project Grant from the Nova Scotia Health Research Foundation. These data form part of a doctoral thesis submitted by E.L.; we are grateful to Drs. Ben Rusak, Ray Klein, and Penny Corkum for their comments on the original thesis. Our sincere gratitude goes to Dr. Sergio Della Sala for his generosity in sharing the test materials of the dual task that he and his colleagues developed. We appreciate the assistance of Dr. Beverly Butler with neuropsychological testing, of Dr. John 
Christie with technical support, of Dr. Shu-fai Cheung with statistical consultation, and of the sleep technologists at the Capital Health Sleep Disorders Laboratory with conducting and scoring sleep studies. E.L. was supported by the Sir Edward Youde Memorial Overseas Fellowship, and G.E. was supported by a Dalhousie Faculty of Medicine Clinical Research Scholar Award. D.M. provides paid service to Vital Aire for reporting level III cardiorespiratory home studies.

\section{REFERENCES}

Alchanatis, M., Zias, N., Deligiorgis, N., Amfilochiou, A., Dionellis, G., \& Orphanidou, D. (2005). Sleep apnea-related cognitive deficits and intelligence: An implication of cognitive reserve theory. Journal of Sleep Research, 14, 69-75.

Aloia, M.S., Arnedt, J.T., Davis, J.D., Riggs, R.L., \& Byrd, D. (2004). Neuropsychological sequelae of obstructive sleep apneahypopnea syndrome: A critical review. Journal of the International Neuropsychological Society, 10, 772-785.

American Psychiatric Association. (2000). Diagnostic and statistical manual of mental disorders (4th ed., Text Revision). Washington, DC: American Psychiatric Association.

Baddeley, A.D. (1996a). Exploring the central executive. The Quarterly Journal of Experimental Psychology, 49A, 5-28. doi: 10.1080/713755608

Baddeley, A.D. (1996b). The fractionation of working memory. Proceedings of the National Academy of Sciences of the United States of America, 93, 13468-13472. doi: 10.1073/pnas.93.24.13468

Baddeley, A.D. (2003). Working memory: Looking back and looking forward. Nature Reviews Neuroscience, 4, 829-839.

Baddeley, A.D., \& Della Sala, S. (1996). Working memory and executive control. Philosophical Transactions of the Royal Society of London. Series B, Biological Sciences, 351, 1397-1404.

Barnes, M., Houston, D., Worsnop, C.J., Neill, A.M., Mykytyn, I.J., Kay, A., et al. (2002). A randomized controlled trial of continuous positive airway pressure in mild obstructive sleep apnea. American Journal of Respiratory Critical Care Medicine, 165, 773-780.

Bédard, M.A., Montplaisir, J., Malo, J., Richer, F., \& Rouleau, I. (1993). Persistent neuropsychological deficits and vigilance impairments in sleep apnea syndrome after treatment with continuous positive airway pressure (CPAP). Journal of Clinical and Experimental Neuropsychology, 15, 330-341. doi: 10.1080/01688639308402567

Beebe, D.W., \& Gozal, D. (2002). Obstructive sleep apnea and the prefrontal cortex: Towards a comprehensive model linking nocturnal upper airway obstruction to daytime cognitive and behavioral deficits. Journal of Sleep Research, 11, 1-16. doi: 10.1046/j.1365-2869.2002.00289.x

Bonnet, M.H. (1993). Vigilance/sleepiness sequelae of sleep disordered breathing and sleep apnea: Cognitive effects of sleep and sleep fragmentation. Sleep, 16, S65-S67.

Buysse, D.J., Reynolds, C.F., III, Monk, T.H., Berman, S.R., \& Kupfer, D.J. (1989). The Pittsburgh Sleep Quality Index: A new instrument for psychiatric practice and research. Psychiatry Research, 28, 193-213. doi: 10.1016/0165-1781(89)90047-4

Coltheart, M. (1981a). MRC psycholinguistic database user manual: Version 1, from http://www.psych.rl.ac.uk/User_Manual_v1_0.html

Coltheart, M. (1981b). The MRC psycholinguistic database. Quarterly Journal of Experimental Psychology, 33(A), 497-505.

Daneman, M., \& Carpenter, P.A. (1980). Individual differences in working memory and reading. Journal of Verbal Learning and Verbal Behavior, 19, 450-466.
Décary, A., Rouleau, I., \& Montplaisir, J. (2000). Cognitive deficits associated with sleep apnea syndrome: A proposed neuropsychological test battery. Sleep, 23, 369-381.

Della Sala, S., Baddeley, A., Papagno, C., \& Spinnler, H. (1995). Dual-task paradigm: A means to examine the central executive. In J. Grafman, K.J. Holyoak \& F. Boller (Eds.), Structure and functions of the human frontal cortex (pp. 161-171). New York: Annals of the New York Academy of Sciences.

Douglas, N.J. (1998). Systematic review of the efficacy of CPAP. Thorax, 53, 414-415.

Duff, S.C., \& Logie, R.H. (2001). Processing and storage in working memory span. The Quarterly Journal of Experimental Psychology, 54A, 31-48.

Engleman, H.M., Kingshott, R.N., Martin, S.E., \& Douglas, N.J. (2000). Cognitive function in the sleep apnea/hypopnea syndrome (SAHS). Sleep, 23, S102-S108.

Engleman, H.M., Martin, S.E., Deary, I.J., \& Douglas, N.J. (1994). Effect of continuous positive airway pressure treatment on daytime function in sleep apnoea/Hypopnea syndrome. Lancet, 343, 572-575. doi: 10.1016/S0140-6736(94)91522-9

Engleman, H.M., Martin, S.E., Deary, I.J., \& Douglas, N.J. (1997). Effect of CPAP therapy on daytime function in patients with mild sleep apnoea/hypopnoea syndrome. Thorax, 52, 114-119.

Felver-Grant, J.C., Bruce, A.S., Zimmerman, M., Sweet, L.H., Millman, R.P., \& Aloia, M.S. (2007). Working memory in obstructive sleep apnea: Construct validity and treatment effects. Journal of Clinical Sleep Medicine, 3, 589-594.

Ferini-Strambi, L., Baietto, C., Di Giolia, M.R., Castaldi, P., Castronovo, C., Zucconi, M., et al. (2003). Cognitive dysfunction in patients with obstructive sleep apnea (OSA): Partial reversibility after continuous positive airway pressure (CPAP). Brain Research Bulletin, 61, 87-92. doi: 10.1016/S0361-9230(03)00068-6

Feuerstein, C., Naëgelé, B., Pepin, J.-L., \& Levy, P. (1997). Frontal lobe-related cognitive functions in patients with sleep apnea syndrome before and after treatment. Acta Neurologica Beligica, 97, 96-107.

Gevins, A.S., \& Cutillo, B.A. (1993). Spatiotemporal dynamics of component processes in human working memory. Electroencephalography and Clinical Neurophysiology, 87, 128-143.

Gevins, A., Smith, M.E., Le, J., Leong, H., Bennett, J., Martin, N., et al. (1996). High resolution evoked potential imaging of the cortical dynamics of human working memory. Electroencephalography and Clinical Neurophysiology, 98, 327-348.

Grunstein, R. (2005). Continuous positive airway pressure treatment for obstructive sleep apnea-hypopnea syndrome. In M.H. Kryger, T. Roth \& W.C. Dement (Eds.), Principles and practice of sleep medicine (4th ed., pp. 1066-1080). Philadelphia: Elsevier Saunders.

Hartenbaum, N., Collop, N., Rosen, I.M., Phillips, B., George, C.F., Rowley, J.A., et al. (2006). Sleep apnea and commercial motor vehicle operators: Statement from the joint task force of the American College of Chest Physicians, the American College of Occupational and Environmental Medicine, and the National Sleep Foundation. Chest, 130, 902-905. doi: 10.1378/chest.130.3.902

Johns, M.W. (1991). A new method for measuring daytime sleepiness: The Epworth Sleepiness Scale. Sleep Research, 14, 540-545.

Johns, M.W. (1992). Reliability and Factor Analysis of the Epworth Sleepiness Scale. Sleep Research, 15, 376-381.

Jones, K., \& Harrison, Y. (2001). Frontal lobe function, sleep loss and fragmented sleep. Sleep Medicine Reviews, 5, 463-475. 
Kim, H.C., Young, T., Matthews, C.G., Weber, S.M., Woodard, A.R., \& Palta, M. (1997). Sleep-disordered breathing and neuropsychological deficits. American Journal of Respiratory Critical Care Medicine, 156, 1813-1819.

Kingshott, R.N., Vennelle, M., Hoy, C.J., Engleman, H.M., Deary, I.J., \& Douglas, N.J. (2000). Predictors of improvements in daytime function outcomes with CPAP therapy. American Journal of Respiratory Care Medicine, 161, 866-871.

Kushida, C.A., Littner, M., Morgenthaler, T., Alessi, C., Bailey, D., Coleman, J.J., et al. (2005). Practice parameters for the indications for polysomnography and related procedures: An update for 2005. Sleep, 28, 299-521.

Levine, B., Robertson, I.H., Clare, L., Carter, G., Hong, J., Wilson, B.A., et al. (2000). Rehabilitation of executive functioning: An experimental-clinical validation of goal management training. Journal of the International Neuropsychological Society, 6, 299-312.

Lis, S., Krieger, S., Hennig, D., Christian, R., Kirsch, P., Seeger, W., et al. (2008). Executive function and cognitive subprocesses in patients with obstructive sleep apnoea. Journal of Sleep Research, 17, 271. doi: 10.1111/j.1365-2869.2008.00660.x

Montplaisir, J., Bédard, M.A., Richer, F., \& Rouleau, I. (1992). Neurobehavioral manifestations in obstructive sleep apnea syndrome before and after treatment with continuous positive airway pressure. Sleep, 15(Suppl.), S17-S19.

Naëgelé, B., Launois, S.H., Mazza, S., Feuerstein, C., Pépin, J.-L., \& Lévy, P. (2006). Which memory processes are affected in patients with obstructive sleep apnea? An evaluation of 3 types of memory. Sleep, 29, 533-544.

Naëgelé, B., Pepin, J.L., Levy, P., Bonnet, C., Pellat, J., \& Feuerstein, C. (1998). Cognitive executive dysfunction in patients with obstructive sleep apnea syndrome (OSAS) after CPAP treatment. Sleep, 21, 392-397.

Naëgelé, B., Thouvard, V., Pepin, J.L., Levy, P., Bonnet, C., Perret, J.E., et al. (1995). Deficits of cognitive executive functions in patients with sleep apnea syndrome. Sleep, 18, 43-52.

Patel, S.R., White, D.P., Malhotra, A., Stanchina, M.L., \& Ayas, N.T. (2003). Continuous positive airway pressure therapy for treating sleepiness in a diverse population with obstructive sleep apnea. Archives of Internal Medicine, 163, 565-571. doi: 10.1001/archinte.163.5.565

Rechtschaffen, A., \& Kales, A. (1968). A manual of standardized terminology, techniques and scoring system for sleep stages of human subjects. Los Angeles: UCLA Brain Information Service/ Brain Research Institute.

Salthouse, T.A. (1996). The processing-speed theory of adult age differences in cognition. Psychological Review 103, 403-428. doi: 10.1037/0033-295X.103.3.403
Sánchez, A.I., Buela-Casal, G., Bermúdez, M.P., \& Casas-Maldonado, F. (2001). The effects of continuous positive air pressure treatment on anxiety and depression levels in apnea patients. Psychiatry and Clinical Neuroscience, 55, 641-646.

Sanner, B.M., Klewer, J., Trumm, A., Randerath, W., Kreuzer, I., \& Zidek, W. (2000). Long-term treatment with continuous positive airway pressure improves quality of life in obstructive sleep apnoea syndrome. European Respiratory Journal, 16, 118-122.

Sateia, M.J. (2003). Neuropsychological impairment and quality of life in obstructive sleep apnea. Clinical Chest Medicine, 24, 249-259. doi: 10.1016/S0272-5231(03)00014-5

Saunamäki, T., \& Jehkonen, M. (2007). A review of executive functions in obstructive sleep apnea syndrome. Acta Neurologica Scandinavica, 115, 1-11. doi: 10.1111/j.1600-0404.2006. 00744.x

Smith, E.E., Jonides, J., \& Koeppe, R.A. (1996). Dissociating verbal and spatial working memory using PET. Cerebral Cortex, 6, 11-20. doi: 10.1093/cercor/6.1.11

Smith, E.E., Jonides, J., Koeppe, R.A., Awh, E., Schumacher, E.H., \& Minoshima, S. (1995). Spatial versus object working memory: PET investigations. Journal of Cognitive Neuroscience, 7, 337-356.

Sternberg, S. (1966). High-speed scanning in human memory. Science, 153, 652-654. doi: 10.1126/science.153.3736.652

Sternberg, S. (1969). Memory-scanning: Mental processes revealed by reaction-time experiments. American Scientist, 57, 421-457.

Sturm, W., Willmes, K., Orgass, B., \& Hartje, W. (1997). Do specific attention deficits need specific training? Neuropsychological Rehabilitation, 7, 81-103.

Thomas, R.J., Rosen, B.R., Stern, C.E., Weiss, W., \& Kwong, K.K. (2005). Functional imaging of working memory in obstructive sleep-disordered breathing. Journal of Applied Physiology, 98, 2226-2234. doi: 10.1152/japplphysiol.01225.2004

Verstraeten, E., Cluydts, R., Pevernagie, D., \& Hoffmann, G. (2004). Executive function in sleep apnea: Controlling for attentional capacity in assessing executive attention. Sleep, 27, 685-693.

Weaver, T.E. (2001). Outcome measurement in sleep medicine practice and research. Part 2: Assessment of neurobehavioral performance and mood. Sleep Medicine Reviews, 5, 223-236. doi: 10.1053/smrv.2001.0153

Wechsler, D. (1987). Wechsler memory scale-revised manual. New York: The Psychological Corporation.

Wilson, M.D. (1988). The MRC psycholinguistic database: Machine readable dictionary, Version 2. Behavioural Research Methods, Instruments and Computers, 20, 6-11. 